\title{
Protective effect of tanshinone IIA on rat kidneys during hypothermic preservation
}

\author{
XIAOMING ZHANG, DAQIANG HE, LINHAO XU and SHUCAI LING \\ Department of Anatomy and Cell Biology, School of Medicine, Zhejiang University, Zhejiang 310058, P.R. China
}

Received July 28, 2011; Accepted October 13, 2011

DOI: $10.3892 / \mathrm{mmr} .2011 .639$

\begin{abstract}
Renal preservation is a universal problem since ischemia/reperfusion (I/R) injury remains an unresolved issue during the procedure of renal transplantation. Tanshinone IIA, one of the effective components of the traditional Chinese medicine Danshen, was reported to exhibit a variety of biochemical activities, including protection against I/R injury. Therefore, identifying the specific molecular pathway mediating tanshinone IIA protection of renal preservation would be of great value to the patients concerned. In this study, rats were divided into two groups and the kidneys were isolated and preserved in two solutions separately, one with Celsior solution and the other with tanshinone IIA additionally added to the Celsior solution. The superoxide dismutase (SOD) activity and the quantity of malonaldehyde (MDA) were measured, the expression of $\mathrm{CHOP}$ and caspase- 12 were assessed by immunohistochemistry staining, and real-time quantitative reverse transcription-polymerase chain reaction analysis was performed after 0,24 and $48 \mathrm{~h}$ of preservation. A significant increase in the activities of SOD and a decrease in the quantity of MDA were observed in the kidneys preserved with tanshinone IIA at 24 and $48 \mathrm{~h}(\mathrm{P}<0.01)$. The expression of CHOP and caspase-12 was lower in the kidneys preserved with tanshinone IIA at 24 and $48 \mathrm{~h}$ than that in the kidneys preserved with Celsior solution alone $(\mathrm{P}<0.05)$. The results suggest that the supplementation of tanshinone IIA in standard Celsior solution may significantly improve long-term kidney preservation. Attenuating oxidative stress injury and decreasing endoplasmic reticulum (ER) stress-mediated apoptosis may play a role in the protection of kidney hypothermic preservation.
\end{abstract}

\section{Introduction}

Due to tremendous progress in immunosuppressive therapy and surgical techniques, renal transplantation is the gold

Correspondence to: Dr Shucai Ling, Department of Anatomy and Cell Biology, School of Medicine, Zhejiang University, Zhejiang 310058, P.R. China

E-mail: zxm@zju.edu.cn

Key words: tanshinone IIA, kidney, hypothermic preservation, CHOP, caspase- 12 standard therapy for patients with end-stage renal disease (1). However, problems involving ischemia/reperfusion (I/R) damage remain unresolved since, to date, the procedure of renal transplantation requires the grafted organ to go through phases of cold preservation and warm reperfusion (2). It has been reported that the storage conditions of the donor kidney may affect the consequences of I/R injury (3), and the development of better organ preservation methods is a major target which has attracted scientific consideration.

Recently, increasing evidence suggests that endoplasmic reticulum (ER) stress may play a significant role in mediating allograft injury. Transplanted organs are challenged by various insults, including I/R injury, acute rejection episodes or inflammation, which could cause biological disturbances of ER homeostasis, thus leading to ER stress (4). Emadali et al reported that the expression of $\mathrm{BiP}, \mathrm{CHOP} / \mathrm{GADD} 153$ and GADD34, which are known to be induced specifically upon ER stress, was differentially affected upon I/R, thus suggesting that distinct ER stress responses were triggered during each phase of human liver transplantation (5). Xiang et al suggested that ER stress-induced apoptosis is involved in allogeneic T-cell apoptosis, which affects the cardiac allograft survival outcome (6). CHOP and caspase-12 as the index factors of the ER stress-mediated apoptosis pathway may be novel targets in the prevention and therapy of allograft injury. However, it has yet to be fully studied in the procedures of organ preservation.

Danshen (DS), a dried root of Salvia miltiorrhiza, is a traditional Chinese drug, which has proved to be an effective treatment for diseases such as stroke, myocardial infarction, occlusive vasculitis and atherosclerosis, and has been available as a standardized substance for decades in eastern Asia (7). Extensive studies have proven that tanshinone IIA is one of the most abundant components of DS, exhibiting a variety of biochemical activities, including vasorelaxation, elimination of oxygen-derived free radicals, stabilization of vascular endothelial function and protection against I/R injury (8). Investigators have reported that tanshinone IIA improves the protective effects of University of Wisconsin (UW) solution for skeletal muscle preservation by inhibiting ICAM-1 expression and apoptosis (9). In this study, tanshinone IIA was added to the standard hypothermia preservation solution in order to investigate a possible protective effect of tanshinone IIA during hypothermic preservation and explore the possible mechanisms. 


\section{Materials and methods}

Animals. A total of 48 Sprague-Dawley (SD) male rats were purchased from the Experimental Animal Center of Zhejiang University. All procedures were conducted with the approval of the local animal care committee (under NIH policies).

Experimental groups. A total of 48 male SD rats weighing 220-250 g were randomly divided into two groups: the Celsior group, in which the kidneys were stored in Celsior solution following perfusion, and the tanshinone IIA group, in which the kidneys were stored in Celsior solution containing tanshinone IIA (100 $\mu \mathrm{mol} / 1$; Zhejiang Institute for Drug Control) following perfusion. Each group was further subdivided according to preservation times at 0,24 and $48 \mathrm{~h}$.

Kidney hypothermic preservation. All rats in each of the six groups were anaesthetized by intraperitoneal injection with a lethal dose of Nembutal. The kidneys from each rat were fully exposed and the renal vessels were ligated to block blood supply to the kidneys. The renal artery was cannulated using a Tibbs arterial cannula connected to a 50-ml syringe and was perfused with a $4^{\circ} \mathrm{C}$ Celsior solution $(\mathrm{NaOH} 100 \mathrm{mM}, \mathrm{KCl} 15 \mathrm{mM}$, $\mathrm{MgCl}_{2} 13 \mathrm{mM}, \mathrm{CaCl}_{2} 0.25 \mathrm{mM}$, mannitor $60 \mathrm{mM}$, lactobionate $80 \mathrm{mM}$, histidine $30 \mathrm{mM}$, glutamate $20 \mathrm{mM}$; pH 7.4), or with a $4^{\circ} \mathrm{C}$ Celsior solution containing $30 \mu \mathrm{M}$ DE. Kidneys were perfused until the solution effusing from the renal vein appeared clear. The kidneys were then removed and stored in different preservation solutions for 0,24 or $48 \mathrm{~h}$ at $4^{\circ} \mathrm{C}$.

Superoxide dismutase (SOD) activity and malonaldehyde (MDA) level assay. Following 0,24 or $48 \mathrm{~h}$ at $4^{\circ} \mathrm{C}$ in different preservation solutions, the right kidneys from each rat were preserved in liquid nitrogen to measure the activity of SOD and the quantity of MDA using a UV spectrometer (Bio-Tek Instruments, Winooski, VT, USA). The kidneys were weighed, minced with scissors and homogenized in $10 \%$ tissue homogenate (homogenized for $3 \times 10 \mathrm{sec}$ intervals on ice). Tissue homogenate $(5-10 \mathrm{ml}$ of $10 \%)$ was centrifuged at $1,000 \mathrm{rpm}$ for $10 \mathrm{~min}\left(4^{\circ} \mathrm{C}\right)$ and the supernatant was transferred to a new tube; some supernatant was diluted to a concentration of $0.1 \mathrm{~g} /$ $\mathrm{ml}$ for the SOD assay. A Coomassie Brilliant Blue kit (NanJin JinCheng ShenWu YanJiuSuo, NanJin, China) was used to measure the protein concentration.

The SOD assay kit (NanJin JinCheng ShenWu YanJiuSuo) used the xanthine oxidase method, and the xanthine oxidase assay used a compilation of methods from various sources. Xanthine oxidase catalyzes the oxidation of xanthine to uric acid and in the process generates $\mathrm{O}^{2-}$. The $\mathrm{O}^{2-}$ production oxidizes hydroxylamine to nitrite, then nitrite generates a prunosus color production under the function of a specific chromogenic agent; the prunosus color production was followed spectrophotometrically, allowing for quantitative measurement. For the SOD assay, $20 \mu \mathrm{l}$ of supernatant $(0.1 \mathrm{~g} / \mathrm{ml})$ was added to the reaction system, and this was incubated at $37^{\circ} \mathrm{C}$ for $40 \mathrm{~min}$. Chromogenic agent $(2 \mathrm{ml})$ was added and the mixture was incubated at room temperature for $10 \mathrm{~min}$. The relative absorbance of the supernatants was immediately measured at $550 \mathrm{~nm}$ using a UV spectrometer. $\mathrm{ddH}_{2} \mathrm{O}(20 \mu \mathrm{l})$ was used instead of supernatants as a negative control. A $1 \mathrm{mg}$.prot of the sample solution that established $50 \%$ inhibition was used to determine the SOD unit in an assay solution as 1 unit.

The MDA kit (NanJin JinCheng ShenWu YanJiuSuo) used thio-malonylurea methods. MDA was condensated to thibarbituric acid (TBA) to generate a red color production (a colorimetric reaction), which was followed spectrophotometrically, allowing for quantitative measurement. For the MDA assay, $10 \mu \mathrm{l}$ of supernatant (10\% tissue homogenate) was added to the reagent of the MDA kit. This was incubated at $95^{\circ} \mathrm{C}$ for $40 \mathrm{~min}$, centrifuged at $4,000 \mathrm{rpm}$ for $10 \mathrm{~min}\left(4^{\circ} \mathrm{C}\right)$, cooled and the supernatant was transferred to the cuvette for assay. The absorbance of the supernatants was measured at $532 \mathrm{~nm}$ using a UV spectrometer. Alcohol $(10 \mu \mathrm{l})$ was used instead of the supernatants as a negative control, and the standard substance $(10 \mathrm{nmol} / \mathrm{ml})$ from the MDA kit was used as a positive control.

Immunohistochemistry assay. Following 0,24 or $48 \mathrm{~h}$ at $4^{\circ} \mathrm{C}$ in different preservation solutions, one-half of the left kidneys from each rat was preserved in $4 \%$ paraformaldehyde for $24 \mathrm{~h}$, and the other half was put into liquid nitrogen for assay of real-time quantitative reverse transcription-polymerase chain reaction (RT-PCR). The tissues were embedded in paraffin, and transverse paraffin sections (5-mm) were mounted on silane-coated slides (ten slide series with ten sections per slide). Sections were deparaffinized and rehydrated. The sections were treated for antigen retrieval with $10.2 \mathrm{mmol} / \mathrm{l}$ sodium citrate buffer, $\mathrm{pH} 6.1$, for $20 \mathrm{~min}$ at $95^{\circ} \mathrm{C}$ for immunohistochemistry.

The sections were washed in $0.01 \mathrm{M}$ phosphate-buffered saline (PBS) containing 0.3\% Triton X-100 (pH 7.4; PBS-T), then immersed in $2 \%$ normal goat serum in PBS for $2 \mathrm{~h}$ at $37^{\circ} \mathrm{C}$, incubated overnight at $4^{\circ} \mathrm{C}$ with polyclone $\mathrm{CHOP}$ antibody (1:50; Santa Cruz Biotechnology, Santa Cruz, CA, USA) and polyclone caspase-12 antibody (1:50; Santa Cruz Biotechnology) in PBS containing $1 \%$ bovine serum albumin, and washed in PBS ( $3 \times 5 \mathrm{~min})$, incubated in biotinylated goat-anti-rabbit IgG (1:200; Boster) in PBS for $2 \mathrm{~h}$ at room temperature, washed in PBS-T ( $3 \times 5 \mathrm{~min})$, incubated in avidin-biotin-peroxidase complex solution (ABC; 1:100; Boster) for $2 \mathrm{~h}$ at room temperature, then rinsed again in PBS-T ( $3 \times 5 \mathrm{~min})$. Immunolabeling was visualized with $0.05 \%$ DAB plus $0.3 \% \mathrm{H}_{2} \mathrm{O}_{2}$ in PBS. Following staining, the sections were counterstained by hematoxylin and then dehydrated with ethanol and xylene before coverslipping with Permount. Rat immunoglobulin IgG (1:200; Biomeda Corporation, USA) was used instead of the primary antibody as a negative control.

Real-time quantitative RT-PCR analysis of CHOP and caspase-12. Following 0,24 or $48 \mathrm{~h}$ at $4^{\circ} \mathrm{C}$ in different preservation solutions, the remaining one-half of the left kidney was used for total RNA isolation in RT-PCR analysis, and RNA was isolated from the specimens using the TRIzol reagent kit (Invitrogen, USA) according to the manufacturer's instructions. For reverse transcription, RNA concentration was measured spectrophotometrically and $2 \mu \mathrm{g}$ total RNA was added to the cDNA synthesis reaction system $(20 \mu \mathrm{l})$ on a FTC2000 (Funglyn, Canada). The reaction mixture consisted of $4 \mu \mathrm{l}$ 5X RT-Buffer, $2.5 \mu \mathrm{mol} / 1$ oligo d(T), $5 \mathrm{mmol} / 1 \mathrm{dNTPs}$ and 20 units RNAasin (RNase inhibitor). The hexamers were annealed by incubating the samples at $70^{\circ} \mathrm{C}$ for $5 \mathrm{~min}$. M-MLV 
reverse transcriptase 200 units (Promega, USA) was added, then incubated at $42^{\circ} \mathrm{C}$ for $60 \mathrm{~min}$. The reaction was stopped by heating to $72^{\circ} \mathrm{C}$ for $10 \mathrm{~min}$. For real-time RT-PCR, the reaction mixture $(40 \mu \mathrm{l})$ consisted of $4 \mu \mathrm{l}$ cDNA, $35.2 \mu \mathrm{l} \mathrm{SYBR}{ }^{\circledR}$ Premix Ex Taq ${ }^{\mathrm{TM}}$ (Takara, China), $0.5 \mu 15$ units Taq DNA polymerase and $0.3 \mu \mathrm{l}$ of $20 \mathrm{pmol} / \mu \mathrm{l} \mathrm{CHOP}$ or caspase-12 primer (Invitrogen). The cDNA was denatured by heating at $94^{\circ} \mathrm{C}$ for $3 \mathrm{~min}$. The template was amplified by 40 rounds of PCR (denaturation at $94^{\circ} \mathrm{C}$ for $10 \mathrm{sec}$, annealing at $60^{\circ} \mathrm{C}$ for $30 \mathrm{sec}$, extension at $72^{\circ} \mathrm{C}$ for $30 \mathrm{sec}$ ) prior to measuring fluorescence at $72^{\circ} \mathrm{C}$. Meanwhile, the primers used were: for the housekeeping gene, glyceraldehyde-3-phosphate dehydrogenase (GAPDH), in real-time RT-PCR to amplify GAPDH (forward: 5'-GGTGGACCTCATGGCCTACAT-3', reverse: 5'-GCCTCTCTCTTGCTCTCAGTATCCT-3') as an internal control of CHOP (forward: 5'-CGGAGTGTA CCCAGCACCATCA-3', reverse: 5'-CCCTCTCCTTTGGTC TACCCTCA-3') and caspase-12 (forward: 5'-AGGGAT AGCCACTGCTGATACAGA-3', reverse: 5'-CTGTCTCCA CATGGGCCTTTGTT-3').

Statistical analysis. The sections were examined at x400 magnification, with UTHSCSA Image Tools 3.0 (University of Texas Medical School at San Antonio, TX, USA), and the number and optical density of the CHOP and caspase-12-positive cells were determined. A probability of $95 \%$ was taken to indicate a statistically significant difference. Data are presented as the means $\pm \mathrm{SD}$. We made relative quantitation using the comparative $C_{T}$ method. The $\Delta C_{T}$ value was determined by subtracting the average GAPDH $\mathrm{C}_{\mathrm{T}}$ value from the average CHOP or caspase- $12 \mathrm{C}_{\mathrm{T}}$ value. The calculation of $\Delta \Delta \mathrm{C}_{\mathrm{T}}$ involved substraction of all group's $\Delta \mathrm{C}_{\mathrm{T}}$ by the $\Delta \mathrm{C}_{\mathrm{T}}{ }^{\text {control }}$ calibrator value. CHOP and caspase-12 mRNA level in all groups relative to the control was determined by calculating the formula: $2^{-(\Delta \Delta C T)}$.

\section{Results}

The activity of SOD and the quantity of MDA were measured. There was no significant difference between the Celsior group and tanshinone IIA group at the beginning. Following 24 and $48 \mathrm{~h}$ of preservation, the activity of SOD significantly increased and the quantity of MDA decreased in the tanshinone IIA group compared to the Celsior group $(\mathrm{P}<0.01$, Table I).

The expression of CHOP and caspase-12 was assayed by immunohistochemistry (Figs. 1 and 2). Fig. 1 shows the expression of CHOP in the Celsior and tanshinone IIA groups. CHOP expression was not different between the Celsior and tanshinone IIA groups at $0 \mathrm{~h}$. However, the number of CHOP-positive cells and the optical density were significantly decreased in the tanshinone IIA group following hypothermic preservation for 24 and $48 \mathrm{~h}$ (Table II). Fig. 2 shows the expression of caspase-12 in the Celsior and tanshinone IIA groups. Similarly to CHOP, the number of caspase-12-positive cells and the optical density were deceased in the tanshinone IIA group at 24 and $48 \mathrm{~h}$ of preservation compared to the Celsior group (Table II).

Table III shows the mRNA level of CHOP and caspase-12 assayed by real-time quantitative RT-PCR. The expression of CHOP and caspase-12 mRNA was not different between the
Table I. Activity of SOD and MDA in the rat kidneys at different time periods following hypothermic preservation.

\begin{tabular}{lcc}
\hline Groups & $\begin{array}{c}\text { MDA } \\
\text { (nmol/mg.prot) }\end{array}$ & $\begin{array}{c}\text { SOD } \\
\text { (U/mg.prot) }\end{array}$ \\
\hline Celsior 0 h & $0.65 \pm 0.13$ & $250.21 \pm 35.60$ \\
Tanshinone IIA 0 h & $0.65 \pm 0.12$ & $255.41 \pm 32.62$ \\
Celsior 24 h & $1.32 \pm 0.27$ & $201.52 \pm 28.11$ \\
Tanshinone IIA 24 h & $1.09 \pm 0.22^{\mathrm{b}}$ & $208.23 \pm 29.25^{\mathrm{a}}$ \\
Celsior 48 h & $1.70 \pm 0.25$ & $185.36 \pm 18.31$ \\
Tanshinone IIA 48 h & $1.14 \pm 0.26^{\mathrm{c}}$ & $204.57 \pm 21.15^{\mathrm{c}}$ \\
\hline
\end{tabular}

vs. the Celsior $24 \mathrm{~h}$ group, ${ }^{\mathrm{a}} \mathrm{P}<0.01$ and ${ }^{\mathrm{b}} \mathrm{P}<0.01$; vs. the Celsior $48 \mathrm{~h}$ group, ${ }^{\mathrm{C}} \mathrm{P}<0.01$.

Table II. Comparison of CHOP- and caspase-12-positive cells from the rat kidneys in all of the groups.

\begin{tabular}{lcc}
\hline Groups & $\begin{array}{c}\text { CHOP-positive } \\
\text { cells }\left(/ \mathrm{mm}^{2}\right)\end{array}$ & $\begin{array}{c}\text { Caspase-12-positive } \\
\text { cells }\left(/ \mathrm{mm}^{2}\right)\end{array}$ \\
\hline Celsior 0 h & $3.58 \pm 0.34$ & $2.11 \pm 0.23$ \\
Tanshinone IIA 0 h & $3.56 \pm 0.42$ & $2.06 \pm 0.25$ \\
Celsior 24 h & $7.86 \pm 1.43$ & $5.86 \pm 0.64$ \\
Tanshinone IIA 24 h & $5.68 \pm 1.42^{\mathrm{a}}$ & $4.26 \pm 0.64^{\mathrm{a}}$ \\
Celsior 48 h & $8.96 \pm 1.65$ & $6.75 \pm 1.20$ \\
Tanshinone IIA 48 h & $6.95 \pm 1.57^{\mathrm{b}}$ & $5.10 \pm 0.95^{\mathrm{b}}$ \\
\hline
\end{tabular}

vs. the Celsior $24 \mathrm{~h}$ group, ${ }^{\mathrm{a}} \mathrm{P}<0.05$; vs. the Celsior $48 \mathrm{~h}$ group, ${ }^{\mathrm{b}} \mathrm{P}<0.05$.

Celsior $0 \mathrm{~h}$ and the tanshinone IIA $0 \mathrm{~h}$ groups. CHOP and caspase-12 mRNA levels were up-regulated in the Celsior groups compared to the tanshinone IIA groups at 24 and $48 \mathrm{~h}$, which was consistent with the results of the immunohistochemical staining.

\section{Discussion}

Renal transplantation has been accepted as a successful therapeutic option for patients with end-stage renal failure. However, the persistent shortage of donor kidneys remains a universal problem due to the inclusion of older, marginal and non-heart-beating donor kidney sources in the donor pool at present (10). These kidneys are more susceptible to ischemic damage, leading to delayed graft function or primary non-function, as well as worse graft function and survival. Two storage approaches have been developed: cold static storage and machine pulsatile perfusion. Although hypothermic perfusion was reported to improve outcome following renal transplantations, static cold storage remains the most practiced preservation method in kidney transplantation (11).

The traditional preservation solution is a type of static cold crystalloid solution that contains impermeants to maintain osmoregulation and control edema, reactive oxygen species scavengers to prevent free radical damage, substrates to maintain energy and buffers to prevent acidosis, such as UW solution, 
Table III. Relative quantification of CHOP and caspase- 12 mRNA levels using the comparative $\mathrm{C}_{\mathrm{T}}$ method followed by real-time quantitative RT-PCR.

\begin{tabular}{|c|c|c|c|c|}
\hline \multirow[t]{2}{*}{ Groups } & \multicolumn{2}{|c|}{$\Delta \mathrm{C}_{\mathrm{T}}$} & \multicolumn{2}{|c|}{ Fold difference } \\
\hline & $\mathrm{CHOP}$ & Caspase-12 & CHOP & Caspase-12 \\
\hline Celsior $0 \mathrm{~h}$ & 13.25 & 13.48 & 1.00 & 1.00 \\
\hline Tanshinone IIA $0 \mathrm{~h}$ & 13.21 & 13.60 & 0.97 & 1.09 \\
\hline Celsior $24 \mathrm{~h}$ & 9.65 & 9.92 & 12.12 & 11.79 \\
\hline Tanshinone IIA $24 \mathrm{~h}$ & 10.04 & 10.30 & 9.25 & 9.06 \\
\hline Celsior $48 \mathrm{~h}$ & 9.51 & 9.67 & 13.36 & 14.03 \\
\hline Tanshinone IIA $48 \mathrm{~h}$ & 9.74 & 9.95 & 11.39 & 11.55 \\
\hline
\end{tabular}

$\left.\left(\Delta \Delta \mathrm{C}_{\mathrm{T}}\right)=\Delta \mathrm{C}_{\mathrm{T}}-\Delta \mathrm{C}_{\mathrm{T}(\text { control })}\right)$ Fold difference $=2^{-\left(\Delta \Delta \mathrm{C}_{\mathrm{T}}\right)}$.
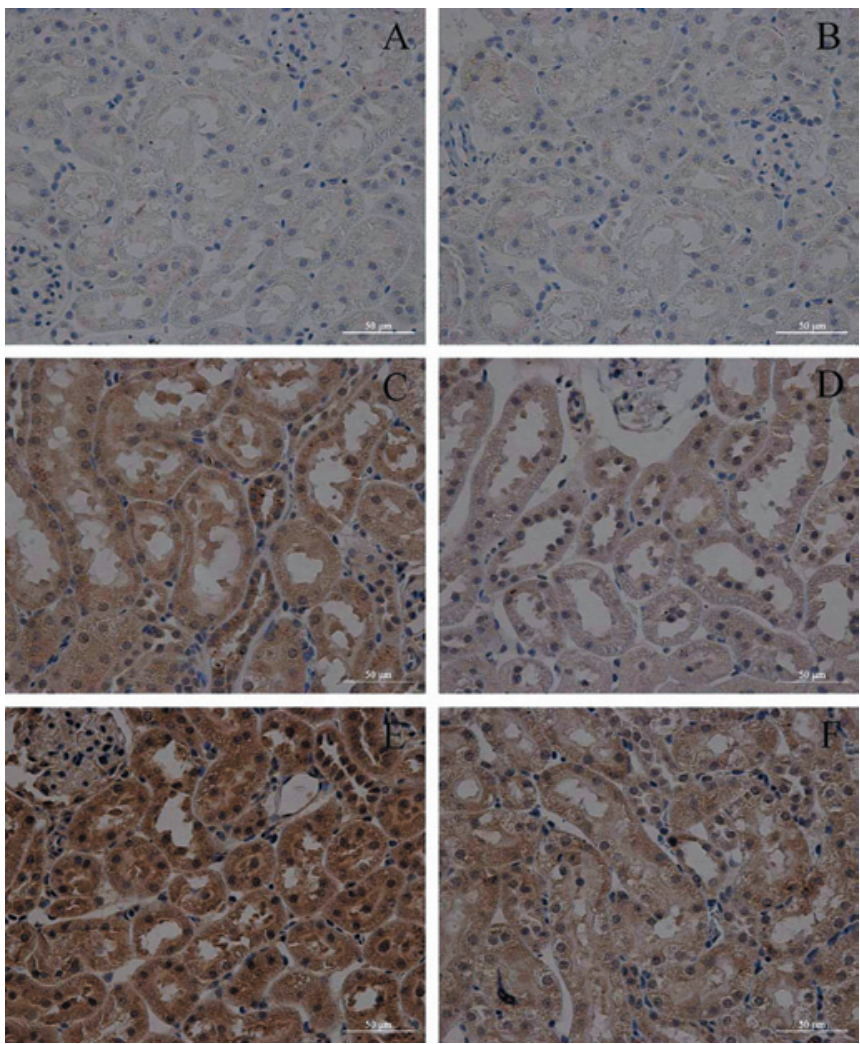

Figure 1. Expression of CHOP detected by immunohistochemistry in rat kidneys following different periods of hypothermic preservation (magnification, $\mathrm{x} 400$ ). (A) Celsior $0 \mathrm{~h}$ group; (B) yanshinone IIA $0 \mathrm{~h}$ group; (C) Celsior $24 \mathrm{~h}$ group; (D) tanshinone IIA $24 \mathrm{~h}$ group; (E) Celsior $48 \mathrm{~h}$ group; (F) tanshinone IIA $48 \mathrm{~h}$ group. Yellow brown granules within the nucleus and cytoplasm were identified as positive CHOP protein staining. Bar, $50 \mu \mathrm{m}$.

which has become a gold standard (12). It has been reported that cold storage is a simple and effective method, which keeps the donor kidney stored for up to $24 \mathrm{~h}$ when the kidney is from a standard deceased donor (13). However, a long-time cold ischemia would be directly related to the severity of $I / R$ injury following transplantation, particularly for allograft retrieved from extended criteria donors (14). I/R has been an inevitable event accompanying kidney transplantation, which may lead to a variety of cellular lesions, including disruption of cell polarization, disassembly of actin cytoskeleton organization,
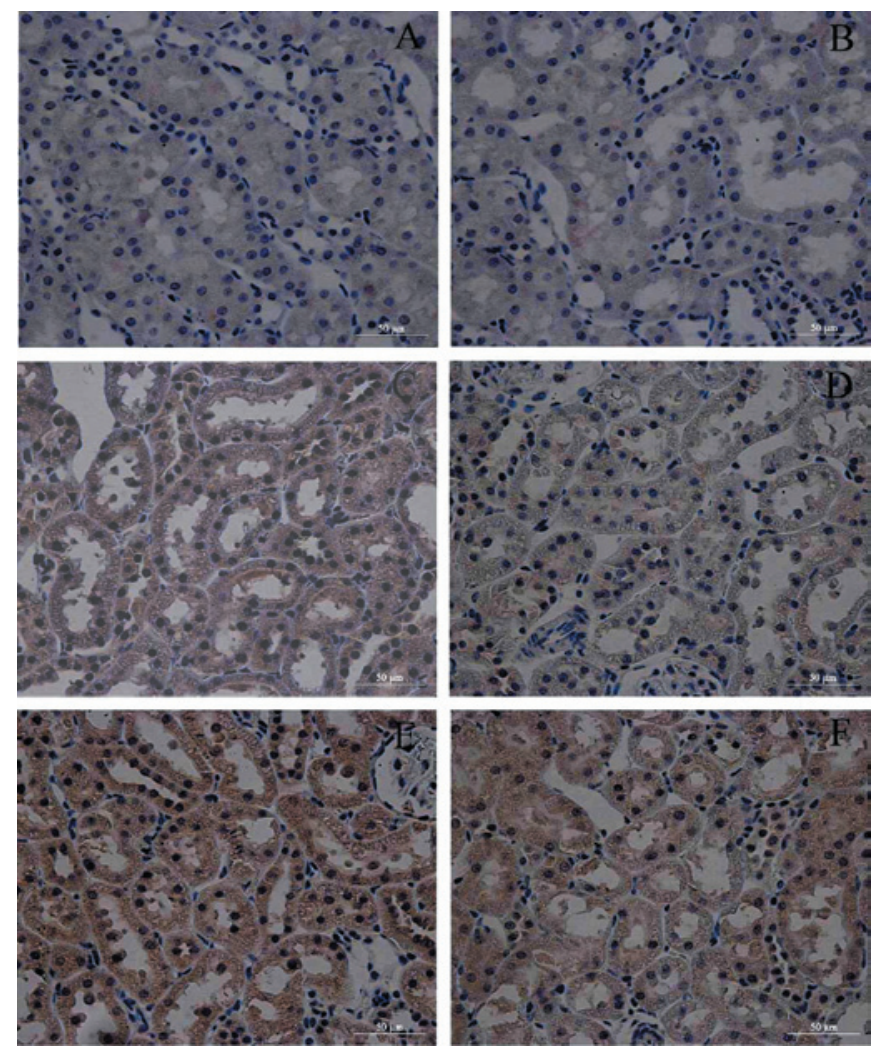

Figure 2. Expression of caspase-12 detected by immunohistochemistry in rat kidneys following different periods of hypothermic preservation (magnification, $\mathrm{x} 400$ ). (A) Celsior $0 \mathrm{~h}$ group; (B) tanshinone IIA $0 \mathrm{~h}$ group; (C) Celsior 24 h group; (D) tanshinone IIA 24 h group; (E) Celsior 48 h group; (F) tanshinone IIA $48 \mathrm{~h}$ group. Yellow brown granules within the cytoplasm were identified as positive caspase-12 protein staining. Bar, $50 \mu \mathrm{m}$.

disruption of tight junctions, necrosis and apoptosis (3). Recent evidence markedly suggests that $\mathrm{I} / \mathrm{R}$ injury may initiate ER stress leading to cellular phenotypic alterations and death (15). ATP deficiency caused by hypoxia and glucose deprivation during ischemia initiates protein misfolding, thereby inducing the unfolded protein response (16). Reperfusion of the affected organ triggers oxidative stress with subsequent alterations of redox-dependent reactions, protein disulfide bond formation and protein folding. CHOP and caspase-12 are two distinct pro-apoptotic transcription factors induced 
uniquely during ER stress. It has been reported that induction of CHOP could be detected between 4 and $8 \mathrm{~h}$ of exposure to ischemia, and pro-caspase-12 was also detectable in the same time frame (17). Minor et al showed significant ultrastructural alterations of ER and marked up-regulation of caspase-12 mRNA following cold storage or oxygenated machine perfusion of rat liver for $18 \mathrm{~h}$, while mitochondrial appearance was unaffected, suggesting the ER to be an early subcellular target of preservation injury (18). Our previous study reported that ER stress was triggered during the hypothermic preservation, and reducing the levels of CHOP and caspase-12 may preserve the donor organ viability (19). Therefore, attempts to improve and prolong preservation conditions with the development of preservation solutions to minimize cold ischemic damage may be an alternative choice (20).

DS has been extensively used in traditional Chinese medicine for decades as an effective remedy for cerebrovascular disorders, angina pectoris and hypertension with only minor side effects (21). Recent medical research has found more than 15 types of chemical structures extracted from DS, and tanshinone IIA was the main effective components of anti-oxidative activity (22), adjustment of the metabolism of blood lipids (23), improvement of rheology, body microcirculation and activity of fibrinolysis (24). Investigators have reported that tanshinone IIA displayed significant protective effects on I/R injury in skeletal muscle preservation (9). In this study, we found that tanshinone IIA down-regulated the quantity of MDA and up-regulated the activity of SOD in preserved renal tissues, which revealed the anti-oxidant activity of tanshinone IIA. Moreover, our histological staining experiments revealed minor pathological destruction of renal tissue in the tanshinone IIA groups compared to the Celsior groups. It clearly showed that tanshinone IIA improved graft survival and reduced renal injury following hypothermic preservation. The mechanism by which tanshinone IIA prolonged kidney allograft survival remains to be determined.

In conclusion, the present study demonstrated that the supplement of tanshinone IIA in standard Celsior solution may significantly improve the long-term kidney preservation consequences by attenuating oxidative stress injury through up-regulation of SOD activity and down-regulation of MDA quantity, and may decrease cell apoptosis by reducing the expression levels of CHOP and caspase-12. The ER stressmediated apoptosis pathway may be a novel target for long-time preservation of donor organs. Further efforts are required to explore the specific mechanism involved.

\section{Acknowledgements}

This study was supported by the National Natural Science Foundation of China (no. 30971124) and the Zhejiang Provincial Natural Science Foundation of China (no. Y2090120).

\section{References}

1. Snoeijs MG, Winkens B, Heemskerk MB, et al: Kidney transplantation from donors after cardiac death: a 25 -year experience. Transplantation 90: 1106-1112, 2010.

2. Haller H, Richter N, Brocker V, Gwinner W, Gueler F and Schwarz A: [Current problems of kidney transplantation] Internist 50: 523-535, 2009.
3. Kosieradzki $\mathrm{M}$ and Rowinski W: Ischemia/reperfusion injury in kidney transplantation: mechanisms and prevention. Transplant Proc 40: 3279-3288, 2008.

4. Pallet N, Fougeray S, Beaune P, Legendre C, Thervet E and Anglicheau D: Endoplasmic reticulum stress: an unrecognized actor in solid organ transplantation. Transplantation 88: 605-613, 2009.

5. Emadali A, Nguyen DT, Rochon C, Tzimas GN, Metrakos PP and Chevet E: Distinct endoplasmic reticulum stress responses are triggered during human liver transplantation. J Pathol 207: 111-118, 2005.

6. Xiang J, Gu X, Qian S and Chen Z: Endoplasmic reticulum stressmediated apoptosis involved in indirect recognition pathway blockade induces long-term heart allograft survival. J Biomed Biotechnol: May, 2010 (E-pub ahead of print).

7. Yu S, Zhong B, Zheng M, Xiao F, Dong Z and Zhang H: The quality of randomized controlled trials on DanShen in the treatment of ischemic vascular disease. J Altern Complement Med 15: 557-565, 2009.

8. Wu YN, Chen Y, Zhang Z, et al: [Study on ultrastructure of cardioprotection of ramipril against ischemia/reperfusion injury in diabetic rats]. Zhongguo Ying Yong Sheng Li Xue Za Zhi 25: 485-489, 2009.

9. Wang HG, Li ZY and Liu XL: Addition of tanshinone IIA to UW solution decreases skeletal muscle ischemia-reperfusion injury. Acta Pharmacol Sin 27: 991-999, 2006.

10. Kootstra G and van Heurn E: Non-heartbeating donation of kidneys for transplantation. Nat Clin Pract Nephrol 3: 154-163, 2007.

11. Yuan X, Theruvath AJ, Ge X, et al: Machine perfusion or cold storage in organ transplantation: indication, mechanisms, and future perspectives. Transpl Int 23: 561-570, 2010.

12. Bond M, Pitt M, Akoh J, Moxham T, Hoyle M and Anderson R: The effectiveness and cost-effectiveness of methods of storing donated kidneys from deceased donors: a systematic review and economic model. Health Technol Assess 13: iii-iv, xi-xiv, 1-156, 2009.

13. Jamieson NV: Kidney preservation times, donor types, and long-term outcomes. Transplantation 83: 255-256, 2007.

14. Hosgood SA, Bagul A and Nicholson ML: Minimising cold ischaemic injury in an experimental model of kidney transplantation. Eur J Clin Invest 41: 233-240, 2010.

15. Bailly-Maitre B, Fondevila C, Kaldas F, et al: Cytoprotective gene bi-1 is required for intrinsic protection from endoplasmic reticulum stress and ischemia-reperfusion injury. Proc Natl Acad Sci USA 103: 2809-2814, 2006.

16. Prachasilchai W, Sonoda H, Yokota-Ikeda N, et al: The protective effect of a newly developed molecular chaperone-inducer against mouse ischemic acute kidney injury. J Pharmacol Sci 109: 311-314, 2009.

17. Szegezdi E, Duffy A, O'Mahoney ME, et al: ER stress contributes to ischemia-induced cardiomyocyte apoptosis. Biochem Biophys Res Commun 349: 1406-1411, 2006.

18. Minor T, Manekeller S, Sioutis M and Dombrowski F: Endoplasmic and vascular surface activation during organ preservation: refining upon the benefits of machine perfusion. Am J Transplant 6: 1355-1366, 2006.

19. Xu L, Han F, Mandal A, Rao GN and Zhang X: Diazoxide attenuates hypothermic preservation-induced renal injury via down-regulation of CHOP and caspase-12. Nephrol Dial Transplant 25: 3859-3867, 2010.

20. Hauet T and Eugene M: A new approach in organ preservation: potential role of new polymers. Kidney Int 74: 998-1003, 2008.

21. Cheng TO: Cardiovascular effects of Danshen. Int J Cardiol 121: 9-22, 2007.

22. Yang R, Liu A, Ma X, Li L, Su D and Liu J: Sodium tanshinone IIA sulfonate protects cardiomyocytes against oxidative stress-mediated apoptosis through inhibiting JNK activation. J Cardiovasc Pharmacol 51: 396-401, 2008.

23. Ji W and Gong BQ: Hypolipidemic activity and mechanism of purified herbal extract of Salvia miltiorrhiza in hyperlipidemic rats. J Ethnopharmacol 119: 291-298, 2008.

24. Han JY,Fan JY, Horie Y, et al: Ameliorating effects of compounds derived from Salvia miltiorrhiza root extract on microcirculatory disturbance and target organ injury by ischemia and reperfusion. Pharmacol Ther 117: 280-295, 2008. 